\title{
THỰC HIỆN TRUY VẤN KHÔNG GIAN VỚI WEBGIS
}

\author{
TRẦN TRỌNG ĐỨC \\ Truòng Đại học Bách Khoa - ĐHQG-HCM
}

\section{Tóm tắt:}

Các ưng dụng WebGIS thể hiện thông tin dạng bản đồ động mà trong đó người dùng có thể thưc hiện các tra xét tìm kiếm thông tin dưa trên những thuộc tính chi định nào đó đã trở nên phổ biến. Tuy nhiên, trong thực tế người dùng có thể có mong muốn thực hiện các tìm kiếm phức tạp hơn, ví dụ nhu tìm kiếm các khách sạn trong vòng bán kính $500 \mathrm{~m}$ tù một vị trí xác định. Các yêu cầu dạng này thuộc vào dạng bài toán truy vấn không gian trong GIS. Nhu là một đóng góp thêm vào trong lĩnh vực này, bài báo trình bày và minh họa một số cách thức thục hiện truy vấn không gian trên Web bao gồm thưc hiện phía Client sủ dụng thu viện JSTS, thục hiện phía server sủ dụng WPS hoặc sủ dụng các hàm phân tích không gian có trong co sở dũ liệu PostgreSQL/PostGIS. Một hệ thống WebGIS - dụa trên các sản phẩm mã nguồn mở Geoserver, và OpenLayers v5.3.0 - đã được xây dụng và được sư dụng để thục hiện minh họa hoạt động truy vấn không gian trong tìm kiếm các đối tượng địa lý trong mối quan hệ không gian với đối tượng địa lý khác. Kết quả thực nghiệm cho thấy việc sủ dụng JSTS hoặc WPS hoặc các hàm không gian trong PostgreSQL/PostGIS để thục hiện truy vấn không gian qua internet là hoàn toàn có thể thực hiện được.

\section{1. Đặt vấn đề}

Việc dễ dàng truy cập vào internet mở ra những cơ hội cho việc chia sẻ thông tin về các đối tượng địa lý (thửa đất, con đường, tuyến cấp nước, thoát nước, ....) phân bố trên một bề mặt địa lý rộng. Do vậy, đã có nhiều phần mềm liên quan đến phục vụ bản đồ và các dịch vụ bản đồ trên Web được các công ty và tổ chức trên thế giới đầu tư phát triển. Chính vì điều này nhiều cơ quan đơn vị trong nước đã bắt đầu tiếp cận với công nghệ thể hiện bản đồ trên Web. Tuy nhiên, các đơn vị, tổ chức ở Việt Nam hiện chỉ khai thác các ứng dụng WebGIS ở khía cạnh hiển thị thông tin dạng bản đồ và thực hiện một số truy vấn tìm kiếm đối tượng địa lý dựa trên một vài những thuộc tính xác định đơn giản. Trong thực tế, người dùng có thể có mong muốn thực hiện các tìm kiếm phức tạp hơn, ví dụ như tìm kiếm các khách sạn trong vòng bán kính $500 \mathrm{~m}$ từ một vị trí xác định hoặc tìm bệnh viện gần nhất đến vị trí đang đứng, ... Các yêu cầu dạng này thuộc vào dạng bài toán truy vấn không gian trong GIS. Tồn tại nhu cầu thực tế như vậy nên nhiều nhóm nghiên cứu hoặc tác giả trên thế giới đã phát triển các hình thức hỗ trợ phân tích hoặc tìm kiếm không gian khác nhau. Nhìn chung các phương pháp truy vấn không gian có thể được sắp xếp vào một trong hai chủng loại hoặc là thực hiện tìm kiếm không gian ngay tại Client hoặc gửi yêu cầu tìm kiếm thông tin về Server để xử lý và sau đó nhận kết quả đã xử lý ở phía Client. Việc chọn phương pháp thực hiện nào sẽ lệ thuộc vào độ phức tạp của phép truy vấn không gian, khả năng xử lý của máy tính tại Client và Server, cũng như phụ thuộc vào thông tin hoặc đối tượng bản đồ cần xử lý có đang thuộc lớp dữ liệu ở định dạng WFS (Web Feature Services) hoặc dữ liệu cần xử lý được lưu trữ tại cơ sở dữ liệu ở Server và tại Client chỉ có dữ liệu được biểu diễn dưới dạng Bitmap WMS (Web Map Services). Trong trường hợp dữ liệu cần truy vấn không gian hiện đang được nạp về Client, tức được thể hiện ở dạng WFS, truy vấn không gian - nếu có thể thực hiện được - nên tiến hành tại Client. Thực hiện truy vấn không gian tại Client có thể được thực hiện với sự hỗ trợ của JavaScript Topology Suit (JSTS) [4]. JSTS là một thư viện JavaScript các hàm không gian 
trong xử lý hình học phù hợp với mô tả "Simple Feature Specification for SQL" được công bố bởi tổ chức Open Geospatial Consortium. JSTS được viết lại một phần dựa vào thư viện nổi tiếng JTS Topology Suite nền Java hỗ trợ việc tạo và làm việc với dữ liệu hình thể [7]. Thư viện Javascript JSTS cần phải được nạp sẵn vào trang WebGIS để làm cơ sở cho việc thực hiện các phép phân tích, tìm kiếm không gian. Thực hiện truy vấn không gian cũng có thể thực hiện tại Map Server với sự hỗ trợ của Web Processing Service (WPS), một chuẩn về xử lý dữ liệu không gian được phát triển bởi tổ chức Open Geospatial Consortium (OGC). OGC WPS định nghĩa một cơ chế, dựa vào đó một ứng dụng ở máy Client có thể gửi một yêu cầu xử lý không gian đến máy Server [9]. Một số dự án mã nguồn mở phát triển thành công, có cung cấp dịch vụ WPS ở phía server, ví dụ Deegree WPS [5], GeoServer WPS [6], và $52^{\circ}$ North WPS [1]. Cần lưu ý rằng, rất nhiều phép phân tích không gian tại Server đòi hỏi phải gửi ngược dữ liệu hình thể của các đối tượng địa lý - đang được biểu diễn ở Client - cần xử lý về cho Map Server. Quá trình chuyển dữ liệu qua lại với khối lượng lớn giữa Client và Server có thể không thực sự hiệu quả về thời gian. Trong trường hợp dữ liệu cần xử lý được lưu trữ tại cơ sở dữ liệu ở Server và tại Client chỉ có dữ liệu được biểu diễn dưới dạng Bitmap WMS, thì chỉ có thể thực hiện các phân tích không gian tại Server. Trong trường hợp này đòi hỏi trước tiên phải sử dụng một số hàm, ví dụ GetFeature của Geoservers, để lấy dữ liệu hình thể cơ bản về Client trước khi gửi ngược dữ liệu về cho Map Server xử lý. Theo [12] việc sử dụng các dịch vụ WPS hoặc sử dụng JSTS để thực hiện xử lý phân tích không gian qua internet là hoàn toàn khả thi. Tuy nhiên, cho đến thời gian gần đây, đã có một số nâng cấp về phía phần mềm, ví dụ Openlayers [10] từ v.2 đã nâng cấp thành v.5.3, đã có một số công nghệ mới được giới thiệu do vậy [12] cần được điều chỉnh và mở rộng. Điểm mở rộng được giới thiệu trong bài báo này là việc sử dụng các truy vấn SQL không gian trong cơ sở dữ liệu không gian PostGIS [11] tại Server. PostGIS là một chương trình phần mềm nguồn mở bổ sung hỗ trợ cho các đối tượng địa lý và các hàm phân tích không gian vào cơ sở dữ liệu quan hệ PostgreSQL. Do tính linh động của việc xây dựng các SQL truy vấn không gian và không có ràng buộc cứng về dữ liệu đầu vào theo quy định bởi WPS, nên hạn chế của việc lấy và truyền dữ liệu về Server để xử lý như trình bày ở trên là có thể khắc phục được.

Tóm lại, trong nghiên cứu này, các cách thức sử dụng Geoserver WPS, PostgreSQL/PostGIS và JSTS trong bài toán tìm kiếm các đối tượng trong mối quan hệ không gian với các đối tượng khác trong một hệ thống WebGIS sẽ được trình bày.

2. Cách thức tiến hành truy vấn không gian trên WebGIS

\subsection{Thục hiện truy vấn không gian với thu viện Javascript JSTS}

Để tiến hành một hoạt động truy vấn không gian nào đó, đòi hỏi 1) phải có dữ liệu hình thể của đối tượng và 2) hàm thực hiện hoạt động truy vấn không gian. Trong hệ thống WebGIS, nếu dữ liệu được nạp về máy tính của Client dưới dạng WFS, thì hình thể của đối tượng có thể lấy được trực tiếp từ các lớp dữ liệu WFS ngay tại Client. Còn hàm thực hiện hoạt động truy vấn không gian sẽ được lấy từ thư viện JSTS. Các hàm của JSTS sẽ được nạp về máy tính người sử dụng nhờ vào địa chỉ khai báo nơi tập tin JavaScript JSTS được lưu trữ, ví dụ $<$ script

$s r c="$ https://unpkg.com/jsts@2.0.2/dist/jsts.min .js " $></$ script $>$ JSTS hỗ trợ các hàm phân tích không gian như tạo vùng đệm (buffer), lấy tâm (centroid), xác định hợp, giao, khác biệt giữa các hình thể... Để thực hiện một hoạt động phân tích không gian tại Client, ví dụ buffer điểm có tọa độ $(0,0)$ với bán kính buffer $=10$, một yêu cầu thực hiện bởi JSTS có dạng như sau:

Var reader $=$ jsts.io. WKTReader();

Var $a$ = reader.read('Point (0 0)');

Var buff = a.buffer(10);

Tất cả các hoạt động xử lý không gian đều 
xảy ra ở máy tính của Client. Các ứng dụng phía Client được thực hiện hầu như chủ yếu dùng JavaScript.

\subsection{Thực hiện truy vấn không gian với WPS}

Vào ngày 13/07/2005, OGC công bố đặc tả dịch vụ Web Processing Service (WPS) hoàn chỉnh đầu tiên - phiên bản 0.4 .0 và phiên bản WPS 1.0.0 được công bố ngày 08/06/2007 đã mở ra khả năng cung cấp, trao đổi và thực hiện công việc xử lý không gian thông qua internet. Đặc tả WPS định nghĩa 3 hoạt động bắt buộc thực hiện bởi một WPS Server, cụ thể là GetCapabilities, DescribeProcess và Execute. Khi sử dụng WPS, người sử dụng có thể đưa ra yêu cầu GetCapabilities để được cung cấp thông tin về các hoạt động xử lý (process) nào có thể có. Để có thông tin chi tiết về yêu cầu dữ liệu nhập và dữ liệu xuất của một WPS process cụ thể, người sử dụng có thể đưa ra yêu cầu DescribeProcess tới WPS Server. Việc thực hiện một WPS process xử lý không gian cụ thể chỉ được tiến hành khi một yêu cầu Execute được gửi đến WPS với tất cả các thông số cần thiết như tên của phép xử lý WPS và các dữ liệu cần được xử lý.

Trong nghiên cứu này WPS Server được sử dụng là module mở rộng WPS của Geoserver. Module này cung cấp một tập hợp các chức năng phân tích không gian dựa trên thư viện của JTS Topology Suite, cũng như một số phép xử lý không gian phát triển bởi GeoServer.

Để thực hiện một hoạt động phân tích không gian tại Server như ví dụ trên, một yêu cầu được gửi tới Map Server, đối với Geoserver thì yêu cầu là: http://localhost:8080/geoserver/wps

Với các tham số được POST dạng XML như sau:

$<? x m l$ version $=$ "1.0" encoding $=$ "UTF8 "? $><$ wps:Execute version $=" 1.0 .0 "$ service $=$ "WPS" ...>

$<$ ows:Identifier $>$ JTS:buffer $</$ ows:Identifier $>$

$<$ wps:Input $>$

$<$ ows:Identifier $>$ geom </ows:Identifier $>$ $<w p s:$ Data $>$

$<$ wps:ComplexData mimeType=" application/wkt" $><!$ [CDATA[Point

0)]] $><$ wps:ComplexData $>$

$</$ wps:Data $>$

$</$ wps:Input $>$

$<$ wps:Input $>$

$<$ ows:Identifier $>$ distance $</$ ows:Identifier $>$ $<$ wps:Data>

$<$ wps:LiteralData $>10</$ wps:LiteralData $>$ $</$ wps:Data $>$

$</$ wps:Input $>$

$</$ wps:DataInputs $><w p s:$ ResponseForm $>$

$<$ wps:RawDataOutput mimeType = "text $/ \mathrm{xml}$; subtype $=$ gml/3.1.1" $>$

$<$ ows:Identifier $>$ result $</$ ows:Identifier $>$

$</$ wps:RawDataOutput $>$

$</$ wps:ResponseForm $>$

$</$ wps:Execute $>$

Việc xây dựng tập tin XML như trên có thể phải chuẩn bị trước với sự hỗ trợ từ $W P S$ Request Builder của Geoserver. Việc xây dựng các XML khá phức tạp đối với người thực hiện WebGIS, các nhà lập trình phát triển của OpenLayers v.2 đã cung cấp đối tượng OpenLayers.WPSClient để giúp tạo ra tập tin XML gửi đến server. Khi đối tượng này được khởi tạo để thực hiện một hoạt động xử lý không gian, người sử dụng cung cấp URL của WPS server và cung cấp các thông số cần thiết như đã mô tả bởi DescribeProcess đến WPS Server, ví dụ cho bài toán buffer ở trên sẽ là 2 thông số

geom: Point $(0,0)$ và dist: 10

OpenLayers.WPSClient sẽ giúp tạo ra tập tin $X M L$ để gưi về Server. Kết quả trả về có dạng, ví dụ POLYGON((10 0, 9.87688 - $1.56434, \ldots$, $9.876881 .56434,100))$

\subsection{Thục hiện truy vấn không gian với PostGIS}

Đối với ví dụ trên, một yêu cầu gửi về cơ sở dữ liệu PostgreSQL/PostGIS phải có dạng ngôn ngữ SQL truy vấn không gian như sau:

Select st_AsText(st_buffer(st_Point $(0,0), 10))$ 
Do không thể kết nối trực tiếp từ trang Web tới cơ sở dữ liệu PostGIS vì lý do an toàn dữ liệu, Node.js [8] là một nền tảng phía Server được xây dựng dựa trên Javascript Engine (V8 Engine), có thể được sử dụng như phần mềm trung gian giúp trao đổi dữ liệu giữa trang Web và cơ sở dữ liệu PostGIS. Cách thức thực hiện như sau: Node.js sau khi nhận được truy vấn từ Web sẽ mở kết nối tới cơ sở dữ liệu PostGIS, thực hiện truy vấn và nhận kết quả trả về. Node.js sau đó sẽ gửi trả kết quả theo yêu cầu về Web dưới dạng, ví dụ GeoJson để có thể hiển thị dưới dạng các đối tượng bản đồ trên trang Web.

\section{Thực nghiệm truy vấn không gian}

Để đánh giá khả năng của việc thực hiện truy vấn dữ liệu không gian thông qua internet, một hệ thống WebGIS đã được xây dựng thử nghiệm và được sử dụng trong phân tích quan hệ không gian giữa các đối tượng thoát nước đang được quản lý như tuyến cống, và hầm ga nằm dọc theo các tuyến cống của một khu vực thuộc thành phố Hồ Chí Minh. Hệ thống WebGIS đã thiết kế hoạt động theo mô hình Client-Server giống như hoạt động của một website thông thường. Hệ thống có kiến trúc 3 tầng (hình 1): Tầng trình bày (Presentation Tier: Client), tầng giao dịch (Bussiness Logic Tier: Application Server), và tầng dĩu liệu (Data Management Tier: Data Server ) [2].

Tầng trình bày gồm một trình duyệt web như Internet Explorer, FireFox, ...dùng để mở các trang Web theo các địa chỉ URL định sẵn. Các trang Web được viết bằng các công nghệ chuẩn của W3C (World Wide Web Consortium) nhằm tăng tính linh động cho web cũng như tăng tính tương tác với người duyệt web. Phần hiển thị, truy vấn thông tin bản đồ trên trang Web được viết sử dụng OpenLayers phiên bản v.5.3.0. Đây là một thư viện JavaScript thuần túy được sử dụng để hiển thị bản đồ theo các chuẩn định dạng $W M S / W F S$ trong hầu hết các trình duyệt Web hiện hành, không lệ thuộc vào phía Server. Tầng giao dịch bao gồm Web Server kết hợp với một ứng dụng bản đồ bên phía server gọi là $M a p$ Server. Trong nghiên cứu này GeoSever được sử dụng như là Map Server. Tầng dũ liệu là nơi lưu trữ các dữ liệu của hệ thống bao gồm dữ liệu không gian và dữ liệu thuộc tính liên quan. (Xem hìn 1)

Dữ liệu thử nghiệm bao gồm dữ liệu nền và dữ liệu chuyên đề về hệ thống thông tin thoát nước được tổ chức thành các lớp dữ liệu và được lưu trong hệ quản trị cơ sở dữ liệu PostgreSQL/PostGIS. Hệ thống WebGIS đã xây dựng có đầy đủ các chức năng cơ bản cần thiết của một hệ thống GIS như tương tác với bản đồ, truy vấn tìm kiếm thông tin (theo một hoặc nhiều tiêu chí), biên tập dữ liệu không gian và ngoài ra còn có nhóm chức năng truy vấn không gian. Trong phạm vi và mục đích của bài báo này, chỉ mô tả cách thức thực hiện truy vấn không gian trong việc chọn, ví dụ các hầm ga nước mưa nằm trong khoảng cách $10 \mathrm{~m}$ so với đối tượng tuyến cống đã được chọn. Trong hệ thống WebGIS, lớp hầm ga được biểu diễn như là một lớp WFS:

var hamga $=$ new VectorLayer $(\{$ title: ' hầm ga',

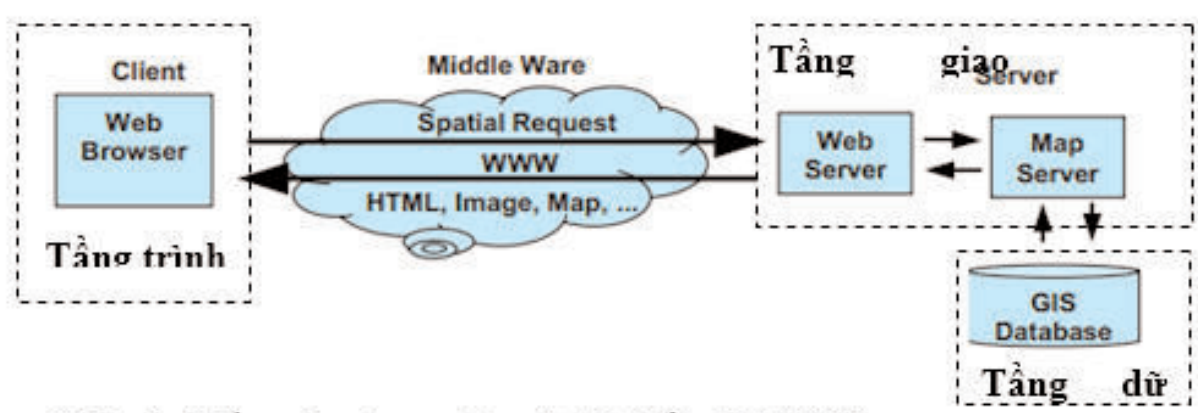

Hình 1. Kiến trúc chung của môt hê thống WebGIS 
source: new VectorSource(\{

format: new GeoJSON(),

loader: function(extent) \{

\$.ajax('http://localhost:8080/geoserver/wfs', \{

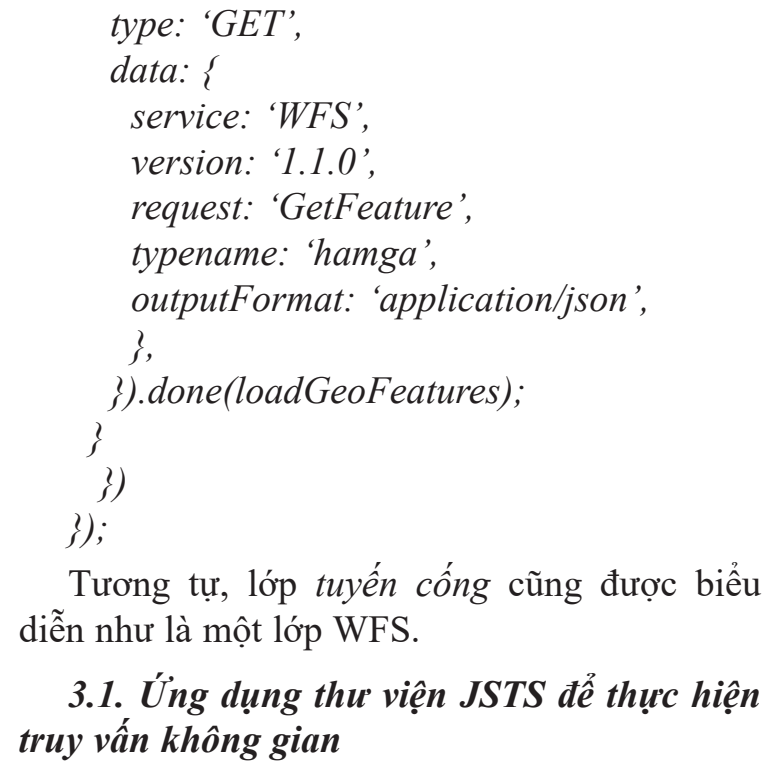

Tương tự, lớp tuyến cống cũng được biểu diễn như là một lớp WFS.

\section{1. Úng dụng thu viện JSTS để thực hiện truy vấn không gian}

Để có thể gọi thực thi các hàm JSTS, đòi hỏi phải nạp vào trang Web thư viện JSTS. Do vậy, đầu tiên phải khởi tạo môi trường làm việc cho hoạt động phân tích không gian bằng cách download về Server và cung cấp thông tin đường dẫn đến thư viện JSTS.

$$
\begin{aligned}
& <s c r i p t \text { type }=\text { "text/javascript" } \\
& \text { src }=\text { "javascript.util.js " }></ \text { script }> \\
& <s c r i p t \text { type }=\text { "text/javascript" } \\
& s r c=\text { "jsts.js } "></ \text { script }>
\end{aligned}
$$

Bước tiếp theo nhằm tạo vùng lân cận $10 \mathrm{~m}$ xung quanh tuyến cống đã chọn. Câu lệnh dùng thực thi hoạt động như dưới đây:

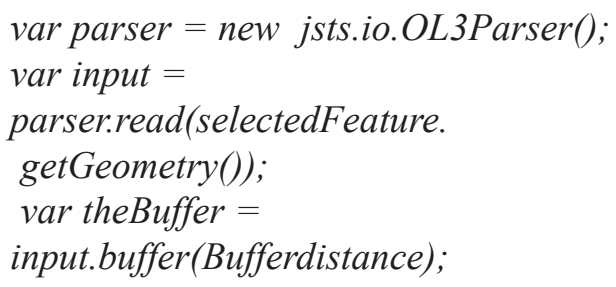

Bước này sử dụng chức năng buffer của thư viện JavaScript JSTS. Các hàm của JSTS, trong đó có hàm buffer, sẽ được nạp về máy tính Client nhờ vào các địa chỉ khai báo nơi tập tin
JavaScript JSTS được lưu trữ. Tuyến cống được chọn là selectedFeature. Hình thể của tuyến cống nhận được nhờ vào selectedFeature. getGeometry(). Kết quả tạo vùng đệm buffer thực hiện hoàn toàn tại Client và sẽ được lưu vào biến theBuffer.

Bước kế tiếp, nhằm tìm kiếm các đối tượng hầm ga giao với vùng đệm $10 \mathrm{~m}$ vừa tạo xung quanh tuyến cống đã chọn. Trong trường hợp lớp hầm ga là lớp dữ liệu có định dạng WFS, bước xử lý có thể thực hiện phía Client với nhóm các câu lệnh sau:

$$
\begin{aligned}
& \text { var fts = hamga.getFeatures(); } \\
& \text { for(var } i=0 ; i<\text { fts.length; } i++)\{ \\
& \text { if (theBuffer.intersects(fts[i]. } \\
& \text { getGeometry()) == true) }\{ \\
& \quad \text { var clone }= \\
& \text { fts }[i] . g e t G e o m e t r y() . c l o n e() ; \\
& \text { var newfeature = new Feature (clone, null); } \\
& \text { highlightLayer.addFeatures(newfeature); } \\
& \quad\{\}
\end{aligned}
$$

Trong nhóm câu lệnh trên, quan hệ hình học giữa vùng đệm xung quanh tuyến cống, theBuffer, với các đối tượng trong lớp tìm kiếm là lớp hầm ga, var fts = hamga.getFeatures() được xác định dựa vào hàm giao intersects. Nếu tồn tại quan hệ intersects giữa theBuffer với đối tượng trong lớp hầm ga thì bản sao của đối tượng hình học này sẽ được đưa vào lớp dữ liệu vector highlightLayer để hiển thị trên bản đồ.

\section{2. Úng dụng thu viện WPS để thục hiện truy vấn không gian}

Đối với bài toán trên, cách thức thực thi trong trường hợp thực hiện truy vấn không gian sử dụng WPS bao gồm hai hoạt động phân tích không gian. Bước đầu tiên là hoạt động tạo vùng đệm 10 m xung quanh tuyến cống đã chọn. Như đã trình bày ở trên để giúp người sử dụng đơn giản trong tạo tập tin XML, OpenLayers v.2 cho phép khởi tạo biến OpenLayers.WPSClient, một giao thức giúp tương tác với Web Processing Services (WPS) và sau đó gọi wpsClient.execute để yêu cầu thục thi 'JTS:buffer'. Trong OpenLayers v.5.3.0 không có tạo sẵn hàm 
OpenLayers.WPSClient do vậy người sử dụng có thể hoặc dựa vào OpenLayers.WPSClient phiên bản v.2 để viết lại wpsClient cho phiên bản v.5.3.0 hoặc dễ dàng hơn nên sử dụng hàm wps.client được giới thiệu bởi [3]. Trong nghiên cứu này, cách thức thực hiện như sau: Tạo biến wpsClient

$$
\text { var wpsClient }=\text { new }
$$

wps.client(\{servers: \{local:

'http://localhost:8080/geoserver/wps' \}\});

Tiếp theo gọi wpsClient.execute để yêu cầu thực thi một hoạt động WPS, trong đó hoạt động xử lý được yêu cầu thực thi là 'JTS:buffer'. Hoạt động xư lý này giúp tạo vùng đệm xung quanh tuyến thoát nước đã chọn.

wpsClient.execute \{

server: 'local', process: 'JTS:buffer',

inputs: \{ geom: selectedFeature, distance: bufferdistance\},

success: function(outputs) \{

theBuffer = outputs.result [0] $\}\})$;

Trong nhóm câu lệnh trên, khoảng cách buffer, gọi là bufferdistance, do người sử dụng cung cấp và trong ví dụ này là $10 \mathrm{~m}$. Tuyến cống được chọn, là selectedFeature. Kết quả tạo vùng đệm sẽ được lưu vào biến theBuffer = outputs.result [0].

Bước kế tiếp, nhằm tìm kiếm các đối tượng hầm ga nằm trong vùng đệm $10 \mathrm{~m}$ xung quanh tuyến cống đã chọn. Bước này được thực hiện như sau:

var intersection $=$

wpsClient.getProcess('local', 'JTS:intersection');

intersection.execute(\{

inputs: \{

a: searchFeatures,

b: outputs.result[0]
\},

success: function(outputs) \{

for (var $i=0, i i=$ outputs.result.length;

$i<i i$; $++i)\{$

highlightLayer.addFeature(outputs.result[i]);

\} 3$\})$;

Thay vì sử dụng các dịch vụ WPS riêng lẻ như ở trên, các dịch dụ WPS có thể được xâu chuổi (chain link) để thực hiện và cho cùng kết quả như sau:

var buffer = wpsClient.getProcess('local', 'JTS:buffer');

buffer.configure $(\{$

inputs: \{

geom: selectedFeature,

distance: bufferdistance $\| 0$

\}));

var intersection $=$

wpsClient.getProcess('local',

'JTS:intersection');

intersection.execute( \{

inputs: \{

a: searchFeatures,

b: buffer.output()

\},

success: function(outputs) \{

for ( $\operatorname{var} \mathrm{i}=0$, $i \mathrm{i}=$ outputs.result.length;

$\mathrm{i}<\mathrm{ii} ;++\mathrm{i})\{$

highlightLayer.addFeature(outputs.result[i]);

\} \}\});

3.3. Úng dụng PostGIS để thục hiện truy vấn không gian

Đối với bài toán trên, cách thức thực thi như sau: 


\author{
\$.ajax(\{ \\ type: "POST", \\ url: \\ "http://localhost:3000/selbyintersection", \\ data: \{id: oid, dist: bufferdistance\}, \\ success: function(response) \{ \\ var data = JSON.parse(response); \\ data.forEach(function (row) \{ \\ var $w k t=$ row.shape; \\ var format $=$ new $W K T()$; \\ var temp $=$ format.readFeature $(w k t,\{\})$; \\ highlightLayer.addFeature(tempfeature); \\ \}); \\ \} \});
}

Như đã trình bày ở trên, do không thể gọi thực thi một câu lệnh SQL trực tiếp từ trang Web tới cơ sở dữ liệu PostGIS, một lệnh gọi được gởi đến node.js Server, cổng 3000 với các tham số oid và bufferdistance. Trong đó oid là id của tuyến cống được chọn.

Tại node.js Server có một hàm tiếp nhận yêu cầu từ client với dạng như sau:

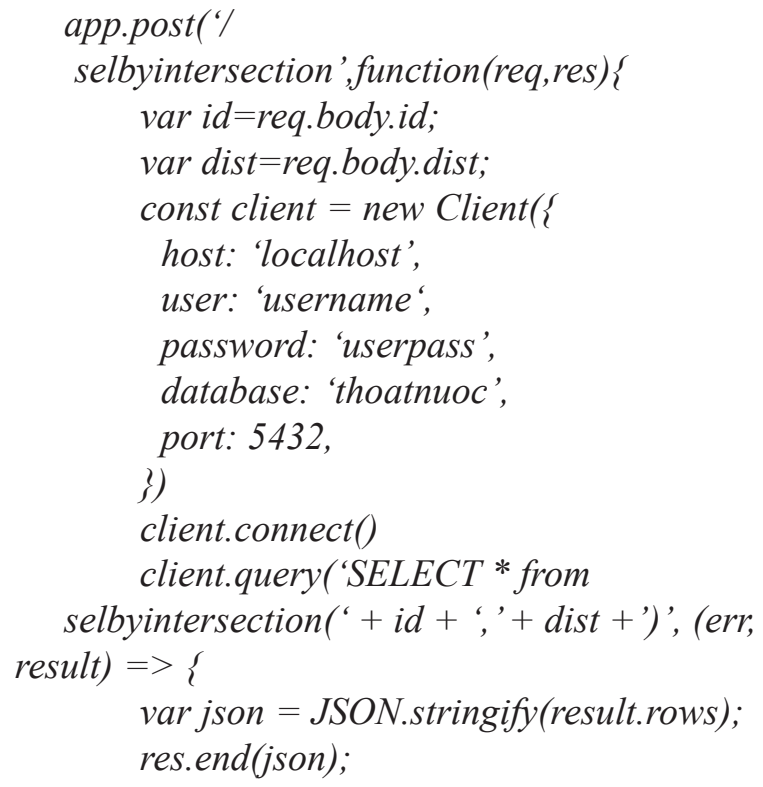

selbyintersection(' + id + ', '+ dist +')', (err, result) $=>\{$

var json = JSON.stringify(result.rows); res.end(json);

client.end();

\})

\});

Selbyintersection là một Store Procedure được thực thi khi nhận được lệnh gọi clien.query(...). Dạng của truy vấn không gian $\mathrm{SQL}$ được lưu trong Store Procedure và được thực thi trong PostGIS có dạng như sau:

select

hamga.objectid,st_AsText(hamga.geom)

from hamga, tuyencong

where tuyencong.objectid $=\mathrm{id}$ and st_intersects(hamga.geom,

$$
\text { st_buffer(tuyencong.geom, dist)); }
$$

U’u điểm của việc thực hiện truy vấn không gian ứng dụng PostGIS so với sử dụng dịch vụ WPS là hạn chế được việc truyền dữ liệu hình thể cần xử lý qua lại giữa Client và Server.

\section{Kết luận}

Tích hợp công nghệ GIS và công nghệ Web mở ra một cơ hội mới không chỉ để chia sẽ, hiển thị và truy vấn thông tin mà còn cho phép thực hiện phân tích không gian thông qua internet. Bài báo này trình bày cách thức như thế nào hoạt động phân tích không gian có thể được thực hiện i) sử dụng thư viện JavaScript JSTS hoặc ii) sử dụng các dịch vụ Web Processing Service (WPS) hoặc iii) sử dụng các hàm phân tích không gian có trong PostGIS. Để minh họa, một hệ thống WebGIS - dựa trên các sản phẩm mã nguồn mở GeoServer, OpenLayers v.5.3... - đã được xây dựng và sau đó được sử dụng để minh họa cách thức tiến hành một hoạt động truy vấn không gian, trong bài báo này là hoạt động tìm kiếm các hầm ga nằm trong vùng đệm $10 \mathrm{~m}$ xung quanh một tuyến cống đã chọn nào đó. Kết quả thực hiện cho thấy việc sử dụng các dịch vụ WPS, PostGIS hoặc sử dụng JSTS để thực hiện xử lý truy vấn không gian qua internet là hoàn toàn khả thi. Việc chọn cách thức thực hiện nào sẽ lệ thuộc vào dữ liệu cần tiến hành phân tích không gian hiện đang được thể hiện ở dạng nào (WFS 
hoặc WMS) tại Client. Nếu dữ liệu đã ở dạng WFS và nếu tồn tại các hàm xử lý không gian cần thiết trong JSTS thì phân tích không gian nên tiến hành tại Client. Còn nếu dữ liệu được thể hiện ở dạng WMS tại Client còn dữ liệu gốc ở Server, thì phân tích không gian nên tiến hành tại Server và nên sử dụng các hàm phân tích không gian của PostGIS do tính linh động của việc xây dựng các $S Q L$ truy vấn không gian và không có ràng buộc cứng về dữ liệu đầu vào theo quy định như của WPS. $\bigcirc$

\section{Tài liệu tham khảo}

[1]. 520 North exploring horizons,

http://52north.org/communities/geoprocessing/, 13 May 2016.

[2]. AA. Alesheikh, H. Helali, HA. Behroz, Web GIS: Technologies and Its Applications, Symposium on Geospatial Theory, Processing and Applications, Ottawa 2002.

[3]. Bart van den Eijnden, Implementing WPS client in OpenLayers 3 ?, https:/gis.stackexchange.com/users/23054/bartvde
[4]. Björn Harrtell, JavaScript Topology Suit (JSTS ), https://github.com/bjornharrtell/jsts.

[5]. Deegree,

http://www.deegree.org/, OSGeo Project, 23 July 2016.

[6]. Geoserver, User Manual,

http://docs.geoserver.org/latest/en/user/, 23 July 2016.

[7]. JTS Topology Suite,

https:/github.com/locationtech/jts

[8]. Node.js, https://nodejs.org/en/

[9]. Open Geospatial Consortium Inc., OpenGIS Web Processing Service, Version 1.0.0, Document number OGC 05-007r7, 2007.

[10]. OpenLayers, User Guide, http://openlayers.org/, 23 July 2016.

[11]. PostGIS, https://postgis.net/

[12]. Trần Trọng Đức, Phân tích không gian trong một hệ thống WEBGIS, Tạp chí Phát triển Khoa học và Công nghệ, T. 19, S. 4K (2016). $O$

\section{Summary}

\section{Implement Spatial Query in WebGIS}

\section{Tran Trong Duc}

\section{Ho Chi Minh city University of Technology, VNU - HCM}

WebGIS applications represent dynamic map information in which users can perform queries on specific attributes that has become popular. However, in practice users may need to do more complex queries, for example searching for hotels within a radius of 500m from a specified location. These requirements belong to category of spatial query in GIS. As an contribution to this aspect, The article presents and illustrates some ways to perform spatial queries on Web, including implementing on Client side using JSTS JavaScript library, implementing on Server side using WPS or using spatial functions in PostgreSQL/PostGIS spatial database. A WebGIS system - based on the open source Geoserver and OpenLayers v5.3.0 - has been developed and used to perform spatial query for features that have spatial relationships with other features. Experiment result shows that the use of JSTS or WPS or spatial functions in PostgreSQL/PostGIS to perform spatial query over the internet is practically doable. $O$ 